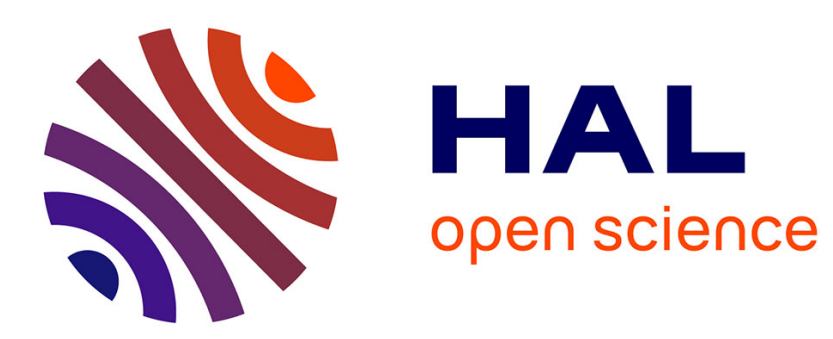

\title{
Processus d'extraction de traits pertinents dans le traitement de données acoustiques
}

\author{
P. Dissard
}

\section{To cite this version:}

P. Dissard. Processus d'extraction de traits pertinents dans le traitement de données acoustiques. Journal de Physique IV Proceedings, 1994, 04 (C5), pp.C5-465-C5-468. 10.1051/jp4:1994597 . jpa00253092

\section{HAL Id: jpa-00253092 https://hal.science/jpa-00253092}

Submitted on 1 Jan 1994

HAL is a multi-disciplinary open access archive for the deposit and dissemination of scientific research documents, whether they are published or not. The documents may come from teaching and research institutions in France or abroad, or from public or private research centers.
L'archive ouverte pluridisciplinaire HAL, est destinée au dépôt et à la diffusion de documents scientifiques de niveau recherche, publiés ou non, émanant des établissements d'enseignement et de recherche français ou étrangers, des laboratoires publics ou privés. 


\title{
Processus d'extraction de traits pertinents dans le traitement de données acoustiques
}

\section{P. DISSARD}

Institut de Psychologie (L.E.A.C.M.), Université Lumière Lyon 2, 5 avenue P. Mendès-France, CP. 11, 69676 Bron cedex, France

\begin{abstract}
This research deals with analogy conversion processes of auditory data into distinctive infra-phonemic unities. These processes have been observed both in tasks of noises verbal categorization and in tasks of word or phoneme recognition in reading with an auditory priming. These conversion processes of acoustic data into phonetic data are in a proeminent position through their influence in the orientation of treatments implicated in these reading tasks. Tasks of lexical decision and phoneme monitoring on a computer in acoustic priming situation (subjects wear a headphone and simultaneously hear a $50 \mathrm{~ms}$ natural noise when a word or pseudo-word appears on the screen), show that analogy conversion processes of pertinent features in the prime noise can guide bottom-up treatments appearing in these experiments (lexical units influence on low levels of target stimuli treatment). Moreover, this extraction is even efficient in an irregular distribution of the acoustic energy (natural noises).
\end{abstract}

Ces travaux décrivent des procédures de conversion de données acoustiques en données infraphonémiques. Ces conversions, orientées par des analogies acoustico-phonétiques, peuvent jouer un rôle dans des tâches impliquant des traitements langagiers (catégorisation verbale de bruits naturels, reconnaissance de mot et détection de phonème - en lecture - en condition d'amorçage auditif).

Il est apparu, dans une tâche de codage de bruits naturels par deux onomatopées de structure CVC et CVCC (une avec comme sommet de syllabe la voyelle [u], l'autre la voyelle [i]), que les sujets orientaient leur choix en fonction d'analogies acoustico-phonétiques entre les bruits et les voyelles centrales des onomatopées (DISSARD \& BANCEL, 1990). Les analogies reposaient sur le timbre. Elles étaient effectuées entre la répartition fréquentielle de l'énergie du bruit et la structure formantique des voyelles $[u]$ et [i]. Ces dernières, se caractérisent et se différencient par un écart minimal entre le $F_{1}$ et le $F_{2}$ pour [u] (cf. fig. 1a) et ce, dans les basses fréquences $\left(F_{1}: 300 \mathrm{~Hz} ; F_{2}: 750 \mathrm{HZ}\right.$ ); par un écart maximal entre le $F_{1}$ et le $F_{2}$ pour [i] (cf. fig. 1 b) et ce, à la fois dans les basses et les hautes fréquences $\left(F_{1}: 300\right.$ $\mathrm{Hz} ; \mathrm{F}_{2}: 2050 \mathrm{~Hz}$ ), (CALLIOPE, 1989).

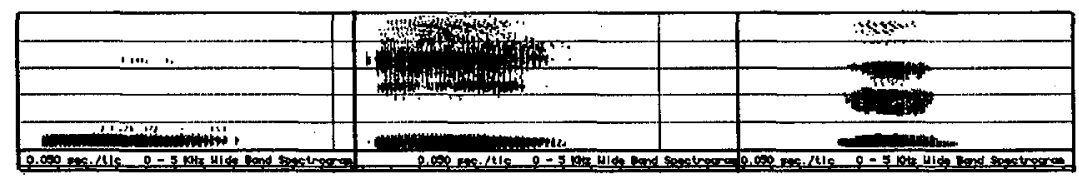

Figure 1: à gauche (a), spectrogramme de la voyelle [u] ; au centre (b), spectrogramme de la voyelle [i], à droite (c), spectrogramme de la voyelle [y] - fréquence d'échantillonnage $10 \mathrm{KHz}$. 
Les vingt bruits ont pu être regroupés en quatre grandes classes, différenciées par le timbre (nous entendons ce terme dans le sens où le définit BOTTE, 1989: "[...] la composition spectrale, plus ou moins riche, des sons complexes ainsi que la distribution de l'énergie dans le spectre déterminent en grande partie la perception du timbre [...]", p.91), et chacune d'entre elles représentaient un type d'analogie, avec un trait phonétique des voyelles [u] et [i], ayant orienté très significativement la catégorisation:

$(\beta)^{*}$ dans le cas où l'énergie n'est présente que dans les hautes fréquences (au-dessus de $\approx 1500 \mathrm{~Hz}$ ), le bruit est associé à l'onomatopée comportant le phonème [i], par analogie avec la présence d'énergie en haute fréquence dans le second formant de [i], mais il y a abstraction de la présence d'énergie en basse fréquence avec le premier formant de cette voyelle; $(\gamma)^{*}$ dans le cas où de l'énergie est présente à la fois dans les hautes et les basses fréquences, plusieurs paramètres secondaires se dessinent : $\left(\gamma^{1}\right)^{*}$ si l'énergie est répartie de façon homogène sur l'ensemble de la bande où elle est présente, le bruit est associé à l'onomatopée comportant le phonème $[\mathrm{u}] ;\left(\gamma^{2}\right) *$ si l'énergie est répartie de façon hétérogène sur la bande (structure formantique du bruit), le bruit est associé à l'onomatopée comportant le phonème [i] sauf, si $\left(\gamma^{3}\right)^{*}$ la (ou les deux) première(s) plage(s) d'intensité marquée est (sont) située(s) dans les fréquences basses ou intermédiaires (entre 800 et $1500 \mathrm{~Hz}$ ) et possède(nt) une énergie nettement plus forte que celle des plages situées dans les hautes fréquences, le bruit étant alors associé à l'onomatopée comportant le phonème $[u]$. Les paramètres complémentaires $\gamma^{1}$ et $\gamma^{2}$ reposent sur une analogie avec la différence d'écart entre les $F_{1}$ et $F_{2}$ des deux voyelles, les deux formants rapprochés de [u] étant alors analysés comme une composante acoustique continue, les deux formants de [i] comme deux plages, la discrimination entre les bruits s'opérant alors selon un critère "une seule plage" versus "plus d'une plage". Le paramètre $\gamma^{3}$ dépendrait des limites fixées aux paramètres $\gamma^{1}$ et $\gamma^{2}$ et de la proximité plus ou moins grande du bruit avec ces limites. Par ailleurs, l'augmentation ou la diminution du taux de catégorisation convergente pour l'une ou l'autre des onomatopées semble être fonction de l'adéquation plus ou moins importante entre le bruit et la représentation "prototypique" de la classe $\left(\beta, \gamma^{1}, \gamma^{2}, \gamma^{3}\right)$ à laquelle il appartient (BEDOIN \& DISSARD, 1992).

Quatre expériences ont été mises en place afin d'étudier plus précisément les processus d'extraction de certaines caractéristiques acoustiques des bruits, sur la base d'analogies acoustico-phonétiques et ce, dans une situation où ils ne peuvent être dirigés attentionnellement. Ces processus ont ainsi été mis en évidence à travers l'observation d'effets d'amorçage, provoqués par le traitement d'un bruit, sur la vitesse de décision lexicale (expériences PL1 \& PL2) et de détection de phonème (expériences PL3 \& PL4). Ces expériences portent sur des mots et pseudo-mots écrits. Il s'agit de situations intermodales, le sujet portant un casque audio et entendant un bruit naturel au moment où le stimulus visuel apparaît. Nous avons contrôlé la présence de certaines analogies acoustico-phonétiques entre bruits-amorces et phonèmes initiaux des stimuli visuels (soit des occlusives - expérience PL1; les voyelles [u] et [y] - expérience PL2; la voyelle [u] - expérience PL3; la voyelle [i] - expérience PL4). Les similitudes acoustiques entre amorces et cibles sont au niveau du timbre. Est donc prise en compte, au niveau des amorces, une caractéristique acoustique globale immédiatement disponible (il s'agit ici de six bruits naturels aux représentations spectrales très différenciées, mais regroupables en deux types de timbre) et, au niveau des cibles, une caractéristique très pertinente dans la discrimination phonétique et plus particulièrement vocalique (répartition formantique). Des variations dans les performances en décision lexicale ou détection de phonème, selon la présence d'analogies acoustico-phonétiques entre amorces et cibles, pourraient ainsi suggérer que certaines informations acoustiques ont été sélectionnées dans le bruit selon des critères "phonétiques".

Description acoustique des bruits amorces: Trois stimuli-amorces, issus de bruits de chocs (B1, B2, $\mathrm{B} 3$ ), ont une répartition homogène de l'énergie des basses fréquences aux hautes fréquences: *très homogène pour $\mathrm{B} 1$ de $200 \mathrm{à} 9500 \mathrm{~Hz}$ (cf. fig. 2a); *très homogène pour $\mathrm{B} 2$ de 100 à $4000 \mathrm{~Hz}$ sur toute la durée du bruit, avec une plage très marquée d'énergie de 4500 à $9500 \mathrm{~Hz}$ sur les vingt premières ms, (cf. fig. 2b); *homogène pour B3 de 100 à $9000 \mathrm{~Hz}$ (cf. fig. 2c).
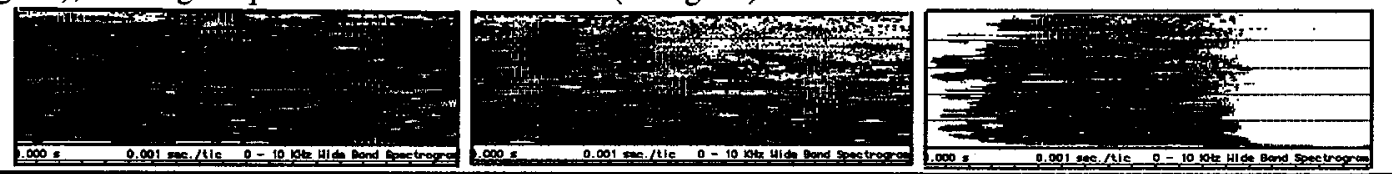

Figure 2: de gauche à droite, (a) spectrogramme du bruit B1, (b) spectrogramme du bruit B2, (c) spectrogramme du bruit $\mathrm{B} 3$, - fréquence d'échantillonnage $20 \mathrm{KHz}$.

Un stimulus amorce (S1) présente une répartition assez homogène de l'énergie de $2000 \mathrm{~Hz}$ à $7000 \mathrm{~Hz}$, de 7000 à $8000 \mathrm{~Hz}$ l'énergie est plus diffuse et il n'y en a pas en moyennes et basses fréquences $(\leq 1500 \mathrm{~Hz})$ (cf. fig. 3a). Un stimulus (S2) présente une prédominance hétérogène de l'énergie de $1500 \mathrm{~Hz}$ à $8000 \mathrm{~Hz}$, 
sa structure est de type "formantique" avec cependant une plage homogène d'énergie, d'intensité marquée, de 4000 à $6000 \mathrm{~Hz}$ (cf. fig. 3b). Un stimulus (S3) présente une répartition hétérogène de l'énergie avec abstraction d'énergie en basses fréquences et une structure acoustique de type "formantique" (deux plages d'énergie très marquées à 2000 et $5500 \mathrm{~Hz}$ ) (cf. fig. 3c).
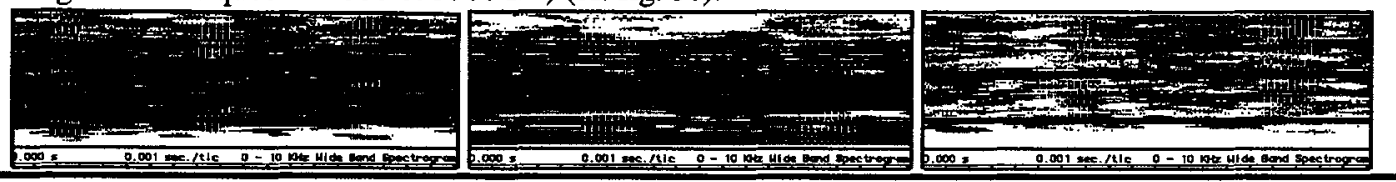

Figure 3: de gauche à droite, (a) spectrogramme du bruit $S 1$, (b) spectrogramme du bruit $S 2$, (c) spectrogramme du bruit $S 3$, - fréquence d'échantillonnage $20 \mathrm{KHz}$.

Description des analogies acoustico-phonétiques entre bruits amorces et caractéristiques phonétiques de certains stimuli-cibles: dans l'expérience PL1, nous étudions *les effets d'amorçage des bruits $\mathrm{B} 1, \mathrm{~B} 2$ et $\mathrm{B} 3$ sur les cibles initialisées par une occlusive, l'analogie reposant sur la structure acoustique homogène de ces trois bruits (cf. fig.2) et le burst (barre d'explosion) se retrouvant dans la catégorie des occlusives. Dans l'expérience PL2, nous étudions *les effets d'amorçage des bruits B1, B2 et B3 sur les cibles initialisées par la voyelle [u], l'analogie reposant sur la structure acoustique homogène de ces trois bruits (cf. fig.2), et le caractère compact de la voyelle [u], (cf. fig.1a); *les effets d'amorçage des bruits $\mathbf{S} 1, \mathrm{~S} 2$ et $\mathrm{S} 3$ sur les cibles initialisées par la voyelle [y], l'analogie reposant sur la structure acoustique hétérogène de ces trois bruits (cf. fig.4), et le caractère diffus de la voyelle [y], (voyelle proche, au niveau phonétique, de [i] - [y]: $F_{1}: 300 \mathrm{~Hz}$ et $F_{2}: 1750 \mathrm{~Hz}$; CALLIOPE, 1989) (cf. fig.1c). Dans l'expérience PL3, nous étudions *les effets d'amorçage du bruit B1 sur les cibles initialisées par la voyelle [u], l'analogie reposant sur la structure acoustique homogène de ce bruit (cf. fig.2a), et le caractère compact de la voyelle [u], (cf. fig.1a). Dans l'expérience PL4, nous étudions *les effets d'amorçage du bruit S3 sur les cibles initialisées par la voyelle [i], l'analogie reposant sur la structure acoustique hétérogène de ce bruit (cf. fig.3c), et le caractère diffus de la voyelle [i], (cf. fig.1b).

Résultats: l'analyse des résultats des quatre expériences révèle des effets d'amorçage en cas d'analogies acoustico-phonétiques entre amorces et cibles. Dans PL1, les amorces homogènes accélèrent la décision lexicale pour les pseudo-mots initialisés par une occlusive. Dans PL2, les amorces homogènes accélèrent la décision lexicale pour les cibles initialisées par $[\mathrm{u}]$ et les amorces hétérogènes accélèrent la décision lexicale pour les cibles initialisées par [y]: ces deux effets sont essentiellement relevés sur les pseudo-mots (DISSARD \& BEDOIN, 1993). Dans PL4, l'amorce hétérogène affecte la détection du phonème [i] à l'initiale des pseudo-mots écrits en retardant la réponse. De même, dans PL3, lorsqu'un pseudo-mot commençant par [u] est associé à un bruit hétérogène (S3), la détection de phonème est plus rapide que lorsqu'il est associé à un bruit homogène (DISSARD \& BEDOIN, 1994).

Description générale de processus d'extraction de traits pertinents dans le traitement des données acoustiques. Les processus d'extraction de traits pertinents, dans des données non-verbales, mis en avant dans PL1 - PL2 - PL3 - PL4, sont orientés par des analogies acoustico-phonétiques. De par les variations de l'effet d'amorçage selon la lexicalité de la cible, des connaissances d'ordre lexical semblent être intervenues dans le processus cognitif orientant ces opérations d'ordre analogique (sinon cet effet serait le même pour les mots et pseudo-mots). Ainsi, les amorces semblent affecter le processus de reconnaissance de mot, ou de détection de phonème, en préactivant certaines représentations de mots, initialisées par un phonème présentant une analogie avec une caractéristique acoustique du bruit. L'activation d'unités lexicales pourrait participer à des étapes très précoces des processus de reconnaissance d'un mot isolé (PL1 et PL2) ou d'identification phonologique (PL3 et PL4), comme le suggèrent les modèles d'activation interactive (Mc CLELLAND \& RUMELHART, 1981 ; RUMELHART \& Mc CLELLAND, 1982). Il semble donc s'opérer une extraction simultanée de traits du bruit et de traits de la cible visuelle qui sont automatiquement confrontés. De cette extraction/confrontation, émergent des caractéristiques communes qui orientent l'activation de représentations lexicales; ces dernières pouvant, par ailleurs, être activées sur une base purement visuelle, lors de la lecture du stimulus cible (cf fig. 4). Les caractéristiques communes entre un bruit et un mot ou pseudo-mot, présenté visuellement, ne peuvent a priori se situer que dans la représentation acoustique du bruit (représentation inhérente au traitement de ce dernier) et dans la représentation phonologique de la cible. Il est cependant à noter que cette explication des processus d'ordre analogique, décrits dans ces expériences, implique l'intervention précoce de traitements phonologiques sur la cible. Il est à signaler que la conception même de ces expérimentations peut être propice à ces derniers, le traitement acoustique du bruit pouvant à lui seul favoriser leur mise en ouvre. De plus, nous nous référons aux travaux de LESCH, M. F., \& POLLATSEK, A. (1993); ces auteurs ayant montré qu'une présentation de $50 \mathrm{~ms}$ (durée retenue pour la présentation de nos stimuli visuels) suffit pour que le codage phonologique d'un mot active des unités lexicales complètes. 


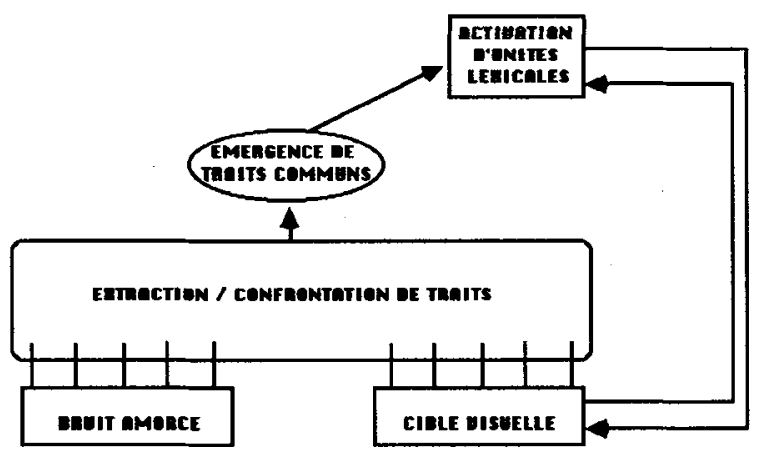

Figure4: Représentation $\left(^{*}\right)$ de l'activation des unités lexicales sur des bases visuelles et/ou par des processus d'extraction/confrontation de traits par analogies acoustico-phonétiques entre amorces et cibles; (*) du rôle des unités lexicales dans les tâches PL1, PL2, PL3 et PL4.

Des différences interviennent dans l'influence de l'amorçage en cas d'analogies acoustico-phonétiques (accélération des temps de réponse pour PL1 et PL2, ralentissement pour PL3 et PL4). Ceci peut s'expliquer par le rôle facilitateur ou non de l'activation lexicale en fonction du contexte. Sous ce terme nous entendons aussi bien la tâche elle-même (décision lexicale ou détection de phonème), que la nature de la cible (mot ou pseudo-mot). Dans PL1 et PL2, les connaissances lexicales préactivées par l'amorçage sont pertinentes par rapport à la tâche (décision lexicale). Elles ont ainsi probablement facilité les traitements des pseudo-mots par une intervention à un niveau précoce (reconnaissance de lettres, de phonèmes ...). Inversement, dans PL3 et PL4, les connaissances lexicales ainsi dégagées ont pu entraîner une surcharge cognitive, leur activation n'étant pas intrinsèquement requise par la tâche (détection de phonème). Ceci pourrait donc expliquer le fait, qu'en cas d'analogie amorce-cible, il y ait un ralentissement dans la réponse, alors qu'en cas d'inadéquation amorce-cible le temps de décision est plus rapide. Dans de tels cas d'inadéquation l'activation lexicale ne peut être que faible car suscitée uniquement par les données visuelles issues du pseudo-mot. Il est cependant à signaler qu'en condition neutre (pas de bruit en amorce), l'activation lexicale devrait être aussi faible qu'en cas d'inadéquation amorce-cible et nous pourrions ainsi nous attendre à des performances égales. Cette attente est confortée dans PL4 mais pas dans PL3 où la condition neutre entraîne un retard, que nous interprétons par l'attente d'un bruit simultané à l'apparition de la cible (et ce, malgré la consigne faisant mention de la possibilité de l'apparition d'une cible sans bruit). Cette interprétation, bien qu'à vérifier, est néanmoins fruit des entretiens post-expérimentaux où nombre de sujets se sont déclarés surpris par l'apparition de la cible, lorsque cette dernière n'était pas couplée à un stimulus auditif.

\section{BIBLIOGRAPHIE}

BEDOIN, N., \& DISSARD, P. (1992) [4]. Quantification de l'Analogie et Concept de Spécicalité. M. Brissaud, M. Grange \& N. Nicoloyannis, Intelligence Artificielle et Sciences Cognitives, 73-91, Hermès, Paris.

BOTTE, M.C. (1989) [3]. L'Audition. Système auditif, perceptions et organisation perceptive élémentaires. Traité de Psychologie Cognitive (Coll.), "Perception, Action, Langage", 83-127. DUNOD, Paris.

CALLIOPE (1989) [2]. La Parole et son Traitement Automatique. MASSON, Paris.

DISSARD, P., \& B ANCEL, P. (1990) [1]. Les Onomatopées ont-elles une Ame ? Etude Psycholinguistique et Phonétique Acoustique sur un Cas de Détermination Analogique dans le Langage. Actes du Colloque SFP, "Sciences Cognitives", Paris, décembre 1990. Publié dans PHOLIA, vol. 5, 91-104, 1990, CRLS, Lyon.

DISSARD, P., \& BEDOIN, N. (1993) [5]. Rôle de l'Analogie et des Traitements Phonologiques dans la Reconnaissance de Mots. Actes du Colloque Congrès Annuel de la SFP, 13-15 mai 1993, Poitiers.

DISSARD, P. \& BEDOIN, N. (1994) [6]. Utilisation de l'analogie dans des tâches de détection de phonèmes en situation intermodale : arguments en faveur des modeles interactifs. Soumis au Comité de Lecture du Congrès "Jeunes Chercheurs" organisé par l'ARC, 23-25 mars 1994, La Motte d'Aveillans.

LESCH, M. F., \& POLLATSEK, A. (1993) [9]. Automatic Access of Semantic Information by Phonological Codes in Visual Word Recognition. Journal of Experimental Psychology: Learning. Memory and Cognition, 19, (2), 285-294.

Mc CLELLAND, J.L., \& RUMELHART, D. E. (1981) [7]. An Interactive Activation Model of Context Effects in Letter Perception : Part 1. An Account of Basic Findings. Psychological Review. Vol. 88, 375-407.

RUMELHART, D. E., \& Mc CLELLAND, J. L. (1982) [8]. An Interactive Activation Model of Context Effects in Letter Perception : Part 2. The Contextual Enhancement Effect and some Tests and Extensions of the Model. Psychological Review, Vol. $89, n^{\circ} 1,60-94$. 